\title{
Article \\ Uncovering the Role of PhzC as DAHP Synthase in Shikimate Pathway of Pseudomonas chlororaphis HT66
}

\author{
Songwei Wang ${ }^{1}$, Dongliang Liu ${ }^{2}$, Muhammad Bilal ${ }^{3}\left(\mathbb{D}\right.$, Wei Wang ${ }^{1}$ and Xuehong Zhang ${ }^{1, *}$ \\ 1 State Key Laboratory of Microbial Metabolism, School of Life Sciences and Biotechnology, \\ Shanghai Jiao Tong University, Shanghai 200031, China; songwei2211@sjtu.edu.cn (S.W.); \\ weiwang100@sjtu.edu.cn (W.W.) \\ 2 CAS Key Laboratory of Computational Biology, Shanghai Institute of Nutrition and Health, \\ University of Chinese Academy of Sciences, Chinese Academy of Sciences, Shanghai 200031, China; \\ liudongliang2018@sibs.ac.cn \\ 3 School of Life Science and Food Engineering, Huaiyin Institute of Technology, Huaian 211600, China; \\ bilaluaf@hotmail.com \\ * Correspondence: xuehzhang@sjtu.edu.cn; Tel.: +86-21-3420-6742
}

check for updates

Citation: Wang, S.; Liu, D.; Bilal, M.; Wang, W.; Zhang, X. Uncovering the Role of PhzC as DAHP Synthase in Shikimate Pathway of Pseudomonas chlororaphis HT66. Biology 2022, 11, 86. https://doi.org/10.3390/ biology11010086

Academic Editor: James White

Received: 8 December 2021

Accepted: 4 January 2022

Published: 6 January 2022

Publisher's Note: MDPI stays neutral with regard to jurisdictional claims in published maps and institutional affiliations.

Copyright: (c) 2022 by the authors Licensee MDPI, Basel, Switzerland. This article is an open access article distributed under the terms and conditions of the Creative Commons Attribution (CC BY) license (https:// creativecommons.org/licenses/by/ $4.0 /$ )
Simple Summary: This study investigated PhzC, one essential 3-Deoxy-D-arabino-heptulosnate-7phosphate (DAHP) synthase that catalyzes the first step of the shikimate pathway in Pseudomonas chlororaphis. We identified and characterized $p h z C$, which is different from the reported DAHP synthase encoding genes aroF, aroG and aroH in E. coli. PhzC accounts for approximately $90 \%$ of the total DAHP synthase activities in P. chlororaphis and it plays the most critical role in four DAHP synthases in the shikimate pathway. Moreover, the results showed that $p h z C$ in P. chlororaphis HT66 is not sensitive to feedback inhibition. This study demonstrated that $\mathrm{PhzC}$ is essential for phenazine-1carboxamide (PCN) biosynthesis without inhibition in feedback by PCN production. It highlighted the importance of PhzC and applying $P$. chlororaphis for shikimate pathway-derived high-value biological production.

Abstract: DAHP synthase catalyzes the first step in the shikimate pathway, deriving the biosynthesis of aromatic amino acids (Trp, Phe and Tyr), phenazine-1-carboxamide, folic acid, and ubiquinone in Pseudomonas chlororaphis. In this study, we identified and characterized one DAHP synthase encoding gene $p h z C$, which differs from the reported DAHP synthase encoding genes aroF, aroG and aroH in $E$. coli. PhzC accounts for approximately $90 \%$ of the total DAHP synthase activities in $P$. chlororaphis HT66 and plays the most critical role in four DAHP synthases in the shikimate pathway. Inactivation of $p h z C$ resulted in the reduction of PCN production by more than $90 \%$, while the absence of genes aroF, aro $\mathrm{G}$ and aroH reduced $\mathrm{PCN}$ yield by less than $15 \%$, and the production of PCN was restored after the complementation of gene $p h z C$. Moreover, the results showed that $p h z C$ in $P$. chlororaphis HT66 is not sensitive to feedback inhibition. This study demonstrated that gene $p h z C$ is essential for PCN biosynthesis. The expression level of both $p h z C$ and $p h z E$ genes are not inhibited in feedback by PCN production due to the absence of a loop region required for allosteric control reaction. This study highlighted the importance of PhzC and applying P. chlororaphis for shikimate pathway-derived high-value biological production.

Keywords: Pseudomonas chlororaphis; PhzC; shikimate pathway; phenazine-1-carboxamide

\section{Introduction}

The shikimate pathway is one of the most studied pathways in E. coli. Glucose and other carbon sources produce phosphoenolpyruvate (PEP) and erythrose-4-phosphate (E4P) through the glycolytic pathway (EPP) and pentose phosphate pathway (PPP), respectively. Both intermediates react to form 3-deoxy-arabino heptanoic acid-7-phosphate (DAHP) by the enzymatic action of DAHP synthase and enter the shikimate pathway to synthesize 
chorismate, which then further synthesizes an extensive range of valuable products through different metabolic routes [1,2]. Eight core enzymes, AroA, AroB, AroC, AroD, AroE, AroF (or isoenzymes AroG, AroH), AroL and AroK are involved in the shikimate pathway (Figure 1), and their biosynthetic mechanisms have already been explored [3,4]. It is the most common metabolic pathway for the biosynthesis of aromatic amino acids in plants, bacteria, and fungi. Microorganisms and plants use the shikimate pathway to synthesize aromatic amino acids and other important metabolites, including salicylic acid, folic acid, vitamin $K$, iron carrier, coenzyme $Q$ and phenazine compounds [4-6]. Moreover, the aromatic amino acids, 4-hydroxybenzoic acid (4-HBA), gentisate, and other intermediates of the shikimate pathway are further derivatized in various metabolic pathways, resulting in the synthesis of more metabolites [7].

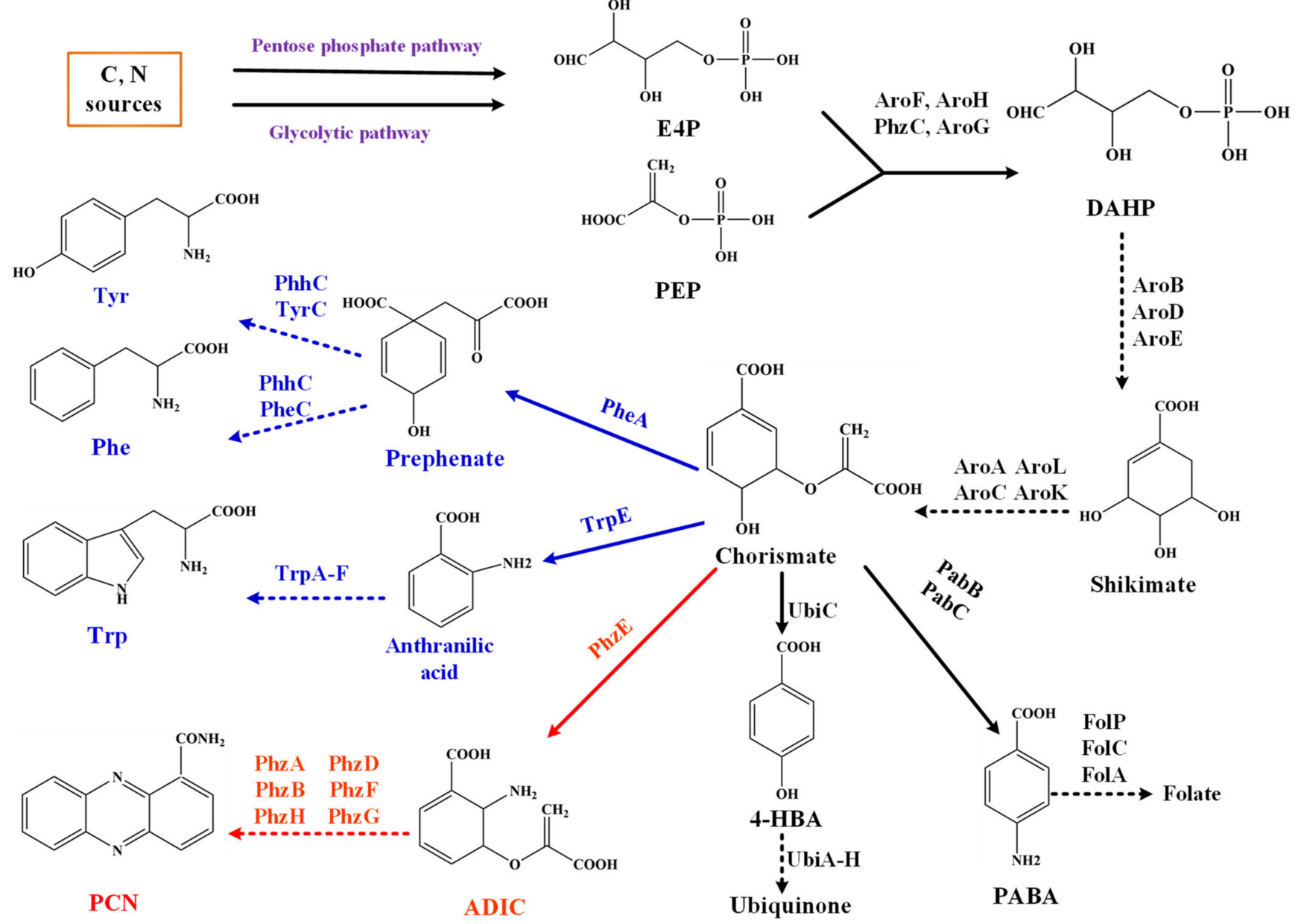

Figure 1. The shikimate pathway and chorismate-derived pathway in P. chlororaphis HT66.

PEP and E4P combine to produce DAHP through a condensation reaction catalyzed by 3-deoxy-arabino heptulose-7-phosphate synthase. The engineered strain expressing an additional copy of the dahp gene showed enhanced glycopeptide production by approximately a factor of three. Deletion of dahp resulted in significant reduction in balhimycin production. Thus, the synthesis of DAHP is a critical step in determining the yield of compounds produced by the shikimate pathway [8].

The shikimate pathway is the leading pathway for the synthesis of numerous aromatic compounds and phenazine antibiotics. The genetically engineered strains for the shikimate pathway have mainly been reported to be Escherichia coli [9], Bacillus subtilis [10], Corynebacterium glutamicum [11], Pseudomonas [7,12] and yeast [13].

The production of shikimate pathway derivatives can be enhanced by increasing precursor supply, blocking the competitive routes and relieving negative regulation. To 
alleviate the stress of feedback inhibition, feedback suppression mutations have been used to enhance the yield of the target compounds. Overexpression of the feedback-suppressor mutant aroG $G^{F B R}$ in C. glutamicum has surged the potential of shikimate pathway and increased the yield of 4-HBA by 21.2-fold [14]. In addition, E. coli DAHP synthases are mainly encoded by aroF and aro $G$, and overexpressing their respective feedback-suppressor mutant aro $G^{F B R}$, aroF ${ }^{F B B R}$ nullifies the effect of feedback inhibition and significantly promotes the yield of shikimate pathway-derived compounds, such as tryptophan, muconic acid [7,15], arbutin [16], maleate [6,17], 2-pyran-4, 6-dicarboxylic acid [18], phenazine-1-carboxylate acid, etc. [19]

Various studies have focused on using E. coli to produce the shikimate pathway products and aromatic amino acids. It has been identified that Pseudomonas spp. synthesizes phenazine compounds through the shikimate pathway (Figure 1). The first systematic analysis of the core phenazine biosynthetic enzymes in Pseudomonas was presented by Floss and coworkers [20]. The sequence analysis of phenazine biosynthetic gene cluster (phz-operon) from $P$. fluorescens 2-79 showed that gene phzC (one of the seven core enzymes involved in phenazine biosynthesis) is not conserved in all phenazine-producing strains. The gene $p h z \mathrm{C}$ is homologous to three isozymes aroF, aroG and aroH. As mentioned earlier, studies explored that the DAHP synthases are sensitive to feedback-inhibition, but a complete sequence analysis of gene $p h z C$ indicated that the enzyme lacks a loop region required for allosteric control function and feedback inhibition [21]. The phzC gene is essential for producing phenazine-1-carboxylic acid (PCA) and generates chorismate by converting carbon metabolites into the shikimate pathway and ensuring sufficient precursor generation [7].

The amino acid sequence analysis of proteins PhzC from P. fluorescens and P. aureofaciens and the DAHP synthases from Streptomyces coelicolor and S. rimosus revealed that these are typical type II enzymes [22]. Since the sequence of DAHP synthases from plants shows a high degree of homology with the indicated microbial DAHP synthases [23,24], plant DAHP synthases differ from bacterial DAHP synthases and are not sensitive to feedback inhibition [25]. Here, neither the sequence of protein nor the location of the gene phzC provides insights into the derivation of the phenazine nucleus [26,27].

Pseudomonas chlororaphis HT66 is a plant growth-promoting rhizobacterium (PGPR) capable of producing PCN with four genes encoding DAHP synthase. This study aims to uncover the essentiality of the DAHP synthase encoding gene $p h z C$ for the shikimate pathway and biosynthesis of phenazine-1-carboxamide (PCN). Pseudomonas chlororaphis HT66 has been studied for many years in our laboratory to synthesize phenazine compounds, such as PCA, PCN, 2-hydroxyphenazine, and 2,3-dihydro-3-hydroxyanthranilic acid (DHHA). These compounds were obtained through metabolic engineering and gene manipulations based on the shikimate pathway [28].

E4P, erythrose-4-phosphate; PEP, phosphoenolpyruvate; DAHP, 3-deoxy-Darabinoheptulosonate7-phosphate; PCN: phenazine-1-carboxamide; 4-HBA, 4-hydroxybenzoic acid; Tyr: tyrosine; Trp: Tryptophan; Phe: Phenylalanine. Main enzymes involved: AroF, AroH, PhzC, AroG: DAHP synthase; PhzE: anthranilate synthase. The phenazine biosynthesis was marked in red, and the aromatic amino acid biosynthesis was marked in blue.

$P$. chlororaphis HT66 can efficiently synthesize phenazine compound PCN, the gene phzC encodes DAHP synthase and is mainly involved in condensing E4P and PEP to form DAHP in P. chlororaphis HT66 (Figure 1). To explore the function of PhzC in the biosynthesis of shikimate pathway derivatives, we explained the effect of gene $p h z C$ on PCN biosynthesis by P. chlororaphis HT66. The analysis of gene phzC function will lay the foundation for the cell factory construction in Pseudomonas based on the shikimate pathway.

\section{Materials and Methods}

\subsection{Bacterial Strains, Plasmids, and Growth Conditions}

All strains used and engineered in this study are listed in Table 1, and the oligonucleotides are summarized in Table 2. Luria-Bertani (LB) medium (Tryptone $10 \mathrm{~g}$, Yeast 
extract $5 \mathrm{~g}, \mathrm{NaCl} 10 \mathrm{~g}, \mathrm{~g} / \mathrm{L})$ was used to incubate $E$. coli and P. chlororaphis during the mutant's construction. E. coli was incubated at $37^{\circ} \mathrm{C}$, while P. chlororaphis was cultured at $28{ }^{\circ} \mathrm{C}$. King's medium B (KB) medium (Glycerol $18 \mathrm{~g}$, Tryptone $20 \mathrm{~g}, \mathrm{MgSO}_{4} 0.732 \mathrm{~g}$, $\mathrm{K}_{2} \mathrm{HPO}_{4} 0.514 \mathrm{~g}, \mathrm{~g} / \mathrm{L}$ ), $\mathrm{K}$ medium (Glycerol $18 \mathrm{~g}, \mathrm{MgSO}_{4} 0.732 \mathrm{~g}, \mathrm{~K}_{2} \mathrm{HPO}_{4} 0.514 \mathrm{~g}$, g/L) and $\mathrm{G}$ medium (Tryptone $20 \mathrm{~g}, \mathrm{MgSO}_{4} 0.732 \mathrm{~g}, \mathrm{~K}_{2} \mathrm{HPO}_{4} 0.514 \mathrm{~g}$, g/L) were used for activating P. chlororaphis for fermentation. Precisely, $100 \mu \mathrm{g} \mathrm{mL} \mathrm{mL}^{-1}$ of ampicillin and $50 \mu \mathrm{g} \mathrm{mL}^{-1}$ of kanamycin were added to the medium for selection.

Table 1. Main strains, plasmids used and developed in this study.

\begin{tabular}{|c|c|c|}
\hline Strains & Description & Source \\
\hline S17-1 ( $\lambda$ pir $)$ & $\begin{array}{l}\text { E. coli res }{ }^{-} \text {pro mod }{ }^{+} \text {integrated copy of } \mathrm{RP}, \mathrm{mob}^{+} \text {, used } \\
\text { for incorporating constructs into } P \text {. chlororaphis }\end{array}$ & Lab stock \\
\hline P. chlororaphis HT66 & P. chlororaphis wild-type, $\mathrm{PCN}, \mathrm{Ap}^{\mathrm{r}}, \mathrm{Sp}^{\mathrm{r}}$ & Lab stock \\
\hline $\mathrm{HT} 66 \Delta p h z E$ & P. chlororaphis HT66 with phzE deleted & This study \\
\hline HT66 $\Delta p h z C$ & P. chlororaphis HT66 with phzC deleted & This study \\
\hline HT66 $\triangle$ aroF & P. chlororaphis HT66 with aroF deleted & This study \\
\hline HT66 $\Delta a r o G$ & P. chlororaphis HT66 with aroG deleted & This study \\
\hline HT66 6 aroH & P. chlororaphis HT66 with aroH deleted & This study \\
\hline HT66 $\Delta p h z C \Delta a r o F$ & P. chlororaphis HT66 with phzC, aroF deleted & This study \\
\hline HT66 $\Delta p h z C \Delta a r o G$ & P. chlororaphis HT66 with phzC, aroG deleted & This study \\
\hline $\mathrm{HT} 66 \Delta p h z \mathrm{C} \Delta a r o H$ & P. chlororaphis HT66 with $\mathrm{phzC}$, aroH deleted & This study \\
\hline 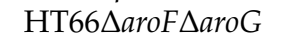 & P. chlororaphis HT66 with aroF, aroG deleted & This study \\
\hline HT66 $\Delta$ aroH $\Delta$ aroG & P. chlororaphis HT66 with aroH, aroG deleted & This study \\
\hline HT66 $\triangle$ aroF $\triangle$ aroH & P. chlororaphis HT66 with aroF, aroH deleted & This study \\
\hline HT66::pBBR & P. chlororaphis HT66 harboring pBBR & This study \\
\hline HT66::phzC & P. chlororaphis HT66 harboring pBBR- $P_{p h z}-p h z C$ & This study \\
\hline HT66::aroF & P. chlororaphis HT66 harboring pBBR-P $p h z^{-a r o F}$ & This study \\
\hline HT66::aroG & P. chlororaphis HT66 harboring pBBR-P $P_{\text {phz }}$-aroG & This study \\
\hline HT66::aroH & P. chlororaphis HT66 harboring pBBR-P phz-aroH & This study \\
\hline HT66 $6 h z C:: p h z C$ & P. chlororaphis HT66 $\mathrm{H}$ h z C harboring pBBR-phzC & This study \\
\hline HT66 6 aroF::aroF & P. chlororaphis HT66 $\triangle$ aroF harboring pBBR-aroF & This study \\
\hline HT66 aroG::aroG & P. chlororaphis HT66 6 aroG harboring pBBR-aroG & This study \\
\hline HT66 $\operatorname{aroH}:: a r o H$ & P. chlororaphis HT66 6 aroH harboring pBBR-aroH & This study \\
\hline Plasmids & Description & Source \\
\hline pk18mobsacB & Broad-host-range gene replacement vector, $\mathrm{Km}^{\mathrm{r}}$ & Lab stock \\
\hline pk18-phzE & pk18mobsacB containing phzE upstream and phzE downstream, $\mathrm{Km}^{\mathrm{r}}$ & This study \\
\hline pk18-phzC & pk18mobsacB containing $p h z C$ upstream and $p h z C$ downstream, $\mathrm{Km}^{\mathrm{r}}$ & This study \\
\hline pk18-aroF & pk18mobsacB containing aroF upstream and aroF downstream, $\mathrm{Km}^{\mathrm{r}}$ & This study \\
\hline pk18-aroG & pk18mobsacB containing aroG upstream and aroG downstream, $\mathrm{Km}^{\mathrm{r}}$ & This study \\
\hline pk18-aroH & pk18mobsacB containing aroH upstream and aroH downstream, $\mathrm{Km}^{\mathrm{r}}$ & This study \\
\hline pBBR-P $P_{p h z}-p h z C$ & pBBR-MCS2 containing $\mathrm{P}_{p h z}-p h z C, \mathrm{Km}^{\mathrm{r}}$ & This study \\
\hline pBBR-P $p h z^{-a r o F}$ & pBBR-MCS2containing $\mathrm{P}_{p h z}-\operatorname{aroF}, \mathrm{Km}^{\mathrm{r}}$ & This study \\
\hline pBBR-P $p h z-a r o G$ & pBBR-MCS2containing $\mathrm{P}_{p h z}$-aroG, $\mathrm{Km}^{\mathrm{r}}$ & This study \\
\hline pBBR-P ${ }_{p h z}-a r o H$ & pBBR-MCS2containing $\mathrm{P}_{p h z}$-aroH, $\mathrm{Km}^{\mathrm{r}}$ & This study \\
\hline
\end{tabular}

For shake-flask fermentation, $P$. chlororaphis and its derivatives were activated at $28{ }^{\circ} \mathrm{C}$ overnight in agar media. A single colony was inoculated in $60 \mathrm{~mL}$ flasks for $12 \mathrm{~h}$ at $28^{\circ} \mathrm{C}$ with $200 \mathrm{rpm}$ shaking, and then $0.6 \mathrm{~mL}$ of the culture was inoculated for fermentation in $60 \mathrm{~mL} \mathrm{~KB}$ medium for $64 \mathrm{~h}$ at $28^{\circ} \mathrm{C}$ with $200 \mathrm{rpm}$.

\subsection{DNA Manipulation and Transformation}

All plasmids used and constructed in this study are listed in Table 1. All genes were amplified with PrimerSTAR Max DNA Polymerase (Takara Bio., Beijing, China). Plasmids containing respective genes were constructed with In-Fusion Cloning Kit (Takara Bio.) Chromosomal in-frame deletions of $\mathrm{phz} C$ were performed separately using the method previously reported [7]. 
Table 2. Main primers designed and used in this study.

\begin{tabular}{|c|c|}
\hline Primer & Sequence $\left(5^{\prime}-3^{\prime}\right)$ \\
\hline & For Gene Deletion \\
\hline phzC-1F & CATGATTACGAATTCACAACTAACCGCTAGCGACACCACT \\
\hline phzC-1R & GATGCGATCACTCTCACGAGAGAATT \\
\hline phzC-2F & TGCGCTTGAACTCAGGAGTCTTTGCCTGGAGTTTGTCGCCATGACCG \\
\hline phzC-2R & GACTCTAGAGGATCCGGTGGAAATCAGTACCCCGACATG \\
\hline aroF-1F & CATGATTACGAATTCAGTTCGATGGCCTCGACGTCTTC \\
\hline aroF-1R & CATGGACTCGGGTGTTTTTTAAGGT \\
\hline aroF-2F & ACСТTAAAAAACACCCGAGTCCATGACCCGTAGCGCTCGATCATCC \\
\hline aroF-2R & GACTCTAGAGGATCCGAAGCAAGCGGCCTATTGCCT \\
\hline aroG-1F & CATGATTACGAATTCACGGTTGCACACTATCAGCCTCG \\
\hline aroG-1R & CGTGTTACTCGTCAGGTCACGGG \\
\hline aroG-2F & CCCGTGACCTGACGAGTAACACGTCCCGTATCGCGGACACAAAA \\
\hline aroG-2R & GACTCTAGAGGATCCGGTGCCAATGGTGCCTACTATTTGA \\
\hline aroH-1F & CATGATTACGAATTCAAATCGCGACAGGATCAGTCCTG \\
\hline aroH-1R & TTCCGCCCCTGTAGGAGCAG \\
\hline aroH-2F & CTGCTCCTACAGGGGCGGAAATTCAAGGCTTCCTGGGCAGG \\
\hline \multirow[t]{2}{*}{ aroH-2R } & GACTCTAGAGGATCCCGTGGCGAGTGTGTCATAAAACCT \\
\hline & For Gene Overexpression \\
\hline G-phzC-1F & TACCGGGCCCCCCCTCGAGTTTGAGCACCACTAAAGTTGAAAACAGG \\
\hline G-phzC-1R & GGCGGCATCCTCCTTAGTTGGG \\
\hline G-phzC-2F & CCCAACTAAGGAGGATGCCGCCATGGAAGACTTACTGAAACGGGTATTAAGTTG \\
\hline G-phzC-2R & TGGCGGCCGCTCTAGATCAAAAGGAGGCAAGGGTTGAGGAG \\
\hline G-aroF-1F & TACCGGGCCCCCCCTCGAGTTTGAGCACCACTAAAGTTGAAAACAGG \\
\hline G-aroF-1R & GGCGGCATCCTCCTTAGTTGGG \\
\hline G-aroF-2F & CCCAACTAAGGAGGATGCCGCCATGATGAGCCAACCCTGGAGCC \\
\hline G-aroF-2R & TGGCGGCCGCTCTAGATCAACGCTTGACCTGTTTCAGGGTC \\
\hline G-aroG-1F & TACCGGGCCCCCCCTCGAGTTTGAGCACCACTAAAGTTGAAAACAGG \\
\hline G-aroG-1R & GGCGGCATCCTCCTTAGTTGGG \\
\hline G-aroG-2F & CCCAACTAAGGAGGATGCCGCCATGGCTGATTTACCGATCAACGACC \\
\hline G-aroG-2R & TGGCGGCCGCTCTAGATCAGGTACGAACCCGTTTTGGCA \\
\hline G-aroH-1F & TACCGGGCCCCCCCTCGAGTTTGAGCACCACTAAAGTTGAAAACAGG \\
\hline G-aroH-1R & GGCGGCATCCTCCTTAGTTGGG \\
\hline G-aroH-2F & CCCAACTAAGGAGGATGCCGCCATGAACTCGTCCGTATCCGCTCTG \\
\hline G-aroH-2R & TGGCGGCCGCTCTAGATCAGGCGGAAGCCGGAATGT \\
\hline
\end{tabular}

Plasmid pBBR1-MCS2 was used to construct the $p h z C$ overexpression strain using native phenazine promoter $\mathrm{P}_{p h z}$. First, gene $p h z C$ and $\mathrm{P}_{p h z}$ promoter from the genomic DNA of $P$. chlororaphis HT66 were amplified. Then, gene $p h z C$ and promoter $\mathrm{P}_{p h z}$ fragments were simultaneously inserted into the plasmid pBBR1-MCS2 using an In-Fusion HD Cloning Kit (Takara Bio.) yielding the plasmid pBBR-P $\mathrm{P}_{\mathrm{i} z}-\mathrm{phz}$. The plasmid was verified through PCR and DNA sequencing before transforming into P. chlororaphis by electroporation (Bio-Rad, Hercules, CA, USA). All of the primers used are shown in Table 2.

\subsection{Sequence and Protein Analysis of DAHP}

DNA sequences of the genes in P. chlororaphis were retrieved from the Pseudomonas Genome Database (http:/ / www.pseudomonas.com/, accessed on 6 December 2019). Sequence homology searching was conducted using the NCBI nucleotide BLAST server. The amino acid sequences of DAHP synthase from other strains were obtained from GenBank. A phylogenetic tree was constructed by MEGA 7.0 using the Neighbor-Joining method.

First, the SWISS-MODEL template library (SMTL version 2020-08-05, PDB release 2020-07-31) [29,30] was searched with BLAST [31] and HHBlits [32] for evolutionary related structures matching the sequence of PhzC. Then, the obtained model was prepared using AutoDock Tools (ADT) [33] and converted into pdbqt mode. Through the site finder function of Molecular Operating Environment (MOE), we predicted a binding site, which 
coincides the positions and reactions mechanism of the two ligands observed in previous studies [34]. Finally, we used MOE to create dummy atoms as the center of the box, and then calculated the affinity of the PhzC with PEP and EFP.

\subsection{Fermentation Process of P. chlororaphis and its Derivative Strains}

$P$. chlororaphis and its derivatives strains were activated overnight at $28{ }^{\circ} \mathrm{C}$ in $\mathrm{KB}$ agar media. Selection of single colonies from Petri plates was performed and used to inoculate approximately $5 \mathrm{~mL}$ of $\mathrm{KB}$ broth in $50 \mathrm{~mL}$ flasks. The primary cultures were incubated overnight at $28{ }^{\circ} \mathrm{C}$ at $200 \mathrm{rpm}$. Portions of these cultures were inoculated into $250 \mathrm{~mL}$ baffled flasks containing $60 \mathrm{~mL} \mathrm{~KB}$ broth to achieve an initial $\mathrm{OD}_{600}$ of 0.02 . The fermentation process was initiated, and the samples were collected every $12 \mathrm{~h}$ to determine cell growth and PCN concentration. When genes were overexpressed in vector pBBR1MCS2, after $6 \mathrm{~h}$ of cultivation, isopropyl $\beta$-D-1-thiogalactopyranoside (IPTG) was added to the culture at a final concentration of $0.1 \mathrm{mM}$. Triplicate experiments were carried out for each fermentation test.

\subsection{Quantitative Real-Time PCR}

$P$. chlororaphis and its derivatives were cultured in KB medium for $26 \mathrm{~h}$, RNA preparation was performed using TaKaRa MiniBEST Universal RNA Extraction Kit. RNA stock was prepared as reported by harvesting cells [7]. Total RNA (500 ng) was immediately used in reverse transcription using the PrimeScript ${ }^{\mathrm{TM}}$ RT reagent Kit. The qPCR reactions were performed in a 96-well plate with a $10 \mu \mathrm{L}$ mixture containing $5 \mu \mathrm{L} 2 \times$ SYBR green ${ }^{\circledR}$ Premix DimerEraser ${ }^{\mathrm{TM}}$ (Takara), $1 \mu \mathrm{L}$ ROX reference dye, $1 \mu \mathrm{L}$ cDNA, and appropriate primer concentration. All samples were analyzed in triplicate. The relative expression levels were calculated based on the reported methods [35]. The rpoD gene encoding sigma factor was used as a reference. The fold change for mRNA was calculated by the $2^{-\Delta \Delta \mathrm{Ct}}$ method. The gene modules were identified using the methods of Fragments Per Kilobase Million (FPKM) [total_exon_fragments/mapped reads (millions) exon length (kB)] [36]. Calculation of the copy numbers of target genes was supplied in supporting information.

\subsection{Quantitative Assay for PCN Production}

To extract PCN, $500 \mu \mathrm{L}$ of fermentation culture was mixed with $25 \mu \mathrm{L}$ of $6 \mathrm{M} \mathrm{HCl}$, and then $3500 \mu \mathrm{L}$ of ethyl acetate was used for extraction. PCN was separated using HPLC (Agilent Technologies 1260 Infinity, Santa Clara, CA, USA) with a C18 reversedphase column $(5 \mu \mathrm{m}, 4.6 \times 12.5 \mathrm{~mm})$ eluted with acetonitrile- $5 \mathrm{mM}$ ammonium acetate $(60: 40, v / v)$. PCN production was quantified using peak area (A) in HPLC elute according to the following formula: PCN $(\mathrm{mg} / \mathrm{L})=0.00871 \mathrm{~A}-3.6617$, which was derived from a dose-peak area plot using purified PCN with a correlation coefficiency $\left(\mathrm{R}^{2}\right)$ of 0.999 . Dry cell weight (DCW) in KB medium was calculated from the optical density at $600 \mathrm{~nm}$ $\left(1_{600}=0.4135 \mathrm{~g} \mathrm{DCW} \mathrm{L}^{-1}\right)$.

\subsection{Statistical Analysis}

The results were averaged and presented as the mean \pm standard deviation from triplicate independent experiments. Significant differences between means $(p<0.05)$ were determined by one-way analysis of variance followed by Duncan's multiple range test (SAS Institute Inc., Cary, NC, USA).

\section{Results}

\subsection{Uncovering DAHP Synthases in P. chlororaphis HT66}

The initial step of the shikimate pathway is the synthesis of DAHP from the precursor's PEP and E4P catalyzed by DAHP synthase (EC 2.5.1.54) [37]. The gene aroG sequence was used against the P. chlororaphis genome in BLAST search analysis. Interestingly, four genes encoding DAHP synthases were found in P. chlororaphis, whereas three DAHP synthase isozymes are harbored in E. coli. One DAHP synthase encoded by gene phzC was only 
found in Pseudomonas. Phylogenetic analysis was carried out to determine the differences between DAHP synthases based on amino acid sequence alignment in $P$. chlororaphis and E. coli. Figure 2 showed that two types of DAHP synthases were identified, four genes encoding DAHP synthase in P. chlororaphis HT66 genomic database are M217_RS0108615 (aroF), M217_RS0108710 (aroG), M217_RS0131975 (aroH) and M217_RS0112890 (phzC). The amino acid sequence of PhzC in P. chlororaphis HT66 is $24 \%, 40 \%$ and $36 \%$, identical to the amino acid sequence of AroF, AroG and AroH of E. coli, respectively. The AroF of P. chlororaphis HT66 is homologous to AroF, AroG and AroH in E. coli with the amino acid sequence at 26\%,29\% and 36\%, respectively. Moreover, AroG amino acid sequence is $60 \%, 54 \%$ and $47 \%$ identical to the amino acid sequences of AroF, AroG and AroH in E. coli, respectively. Similarly, AroH amino acid sequence is 53\%, $49 \%$ and $46 \%$ identical to AroF, AroG and AroH in E. coli, respectively. Sequence analysis of different DAHP synthases showed that DAHP synthases were classified into DAHP synthase type I and DAHP synthase type II, and PhzC from P. chlororaphis HT66 belongs to DAHP synthase type II. Comparative analysis of PhzC in phenazine producing and non-PHZ producing strains was shown in Table S1 in Supplementary File.

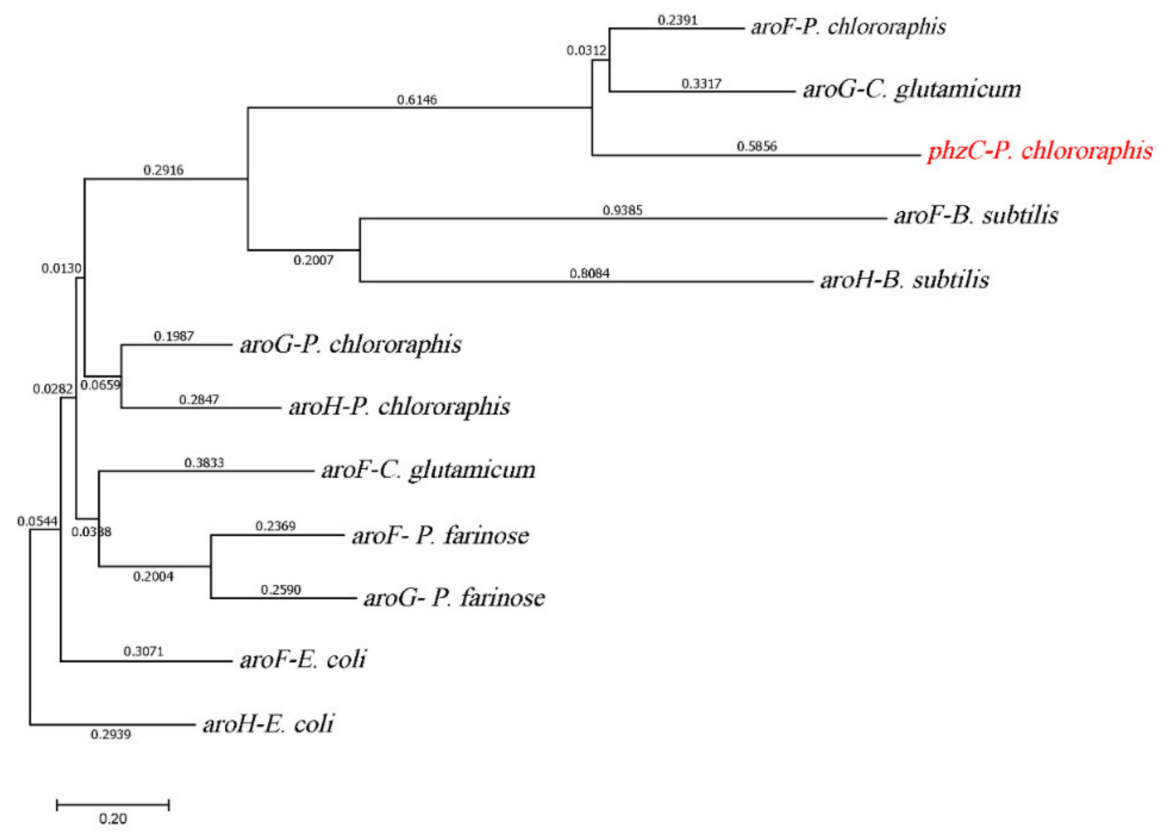

Figure 2. Phylogenetic tree constructed based on the alignment of DAHP synthases.

$p h z C$ encodes the first DAHP synthase in the shikimate pathway, aroF, aroG and aroH encode the next three DAHP synthases in $P$. chlororaphis, whereas only aroF, aroG and $a r o H$ are found in E. coli. Based on our earlier transcriptomic analysis, the FPKM (fragments per kilobase of exon per million fragments mapped) of four DAHP synthases were shown in Figure 3. As illustrated, the FPKM value of gene $p h z C$ was 5590 in the 2hydroxyphenazine-accumulating strain $P$. chlororaphis-AN, which was significantly higher than $\operatorname{aroF}(\mathrm{FPKM}=107)$, aroG $(\mathrm{FPKM}=52)$ and $\operatorname{aroH}(\mathrm{FPKM}=898)$. FPKM values are 108 times, 52 times, and 6 times of that genes aroF, aro $\mathrm{G}$ and $a r o H$, respectively, and it was similar to the previous iTRAQ-based quantitative proteomic analysis [38]. Based on the transcriptomic analysis of phenazine-deficient strain P. chlororaphis-PHZ, no significant change was observed in the transcriptional levels of aroF (FPKM =117), aroG $(\mathrm{FPKM}=52)$, and $\operatorname{aroH}(\mathrm{FPKM}=20)$. Thus, $p h z \mathrm{C}$ is hypothesized to be more essential for the biosynthesis of phenazine derivatives than aroF, aroG, and aroH in P. chlororaphis HT66. 
(A)

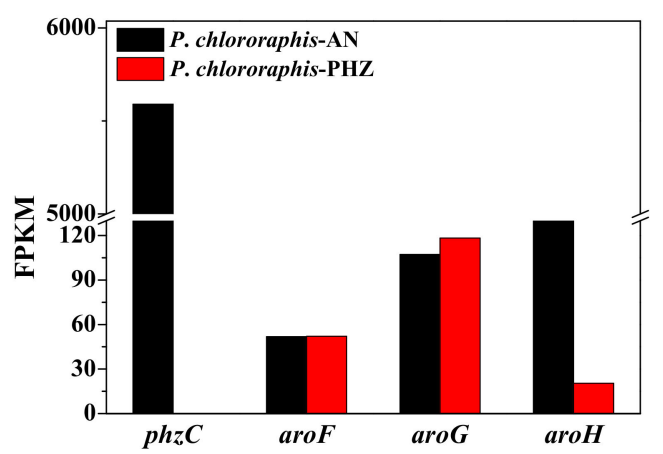

(B)

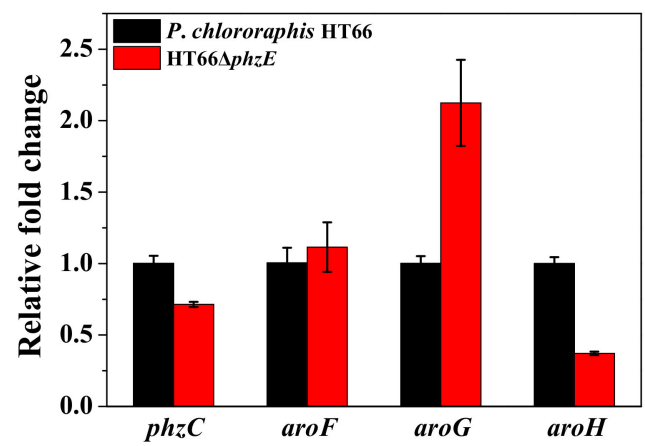

Figure 3. The expression differences of different DAHP synthase enzymes by transcriptome and relative quantitative PCR analysis. (A) Based on transcriptome analysis; (B) Based on relative quantitative PCR analysis.

The P. chlororaphis-AN (with strengthened phenazine synthetic pathway) and P. chlororaphis-AN-PHZ (with inactivated phenazine synthetic pathway) were used for transcriptome analysis.

For further analysis, the PCN biosynthetic pathway was blocked in P. chlororaphis HT66, resulting in phenazine-deficient strain HT66 $\Delta$ phzE. After $26 \mathrm{~h}$ of incubation, the transcription level of different DAHP synthases was noted. Figure $3 \mathrm{~B}$ showed that gene $p h z C$ is down-regulated by 1.4-fold, aroF and aro $G$ are up-regulated by 1.1-fold, 2.1-fold respectively, while gene $a r o H$ is down-regulated by 2.7 -fold after inactivating phenazine synthetic pathway. The transcription level is consistent with both inactivated strains of the entire phenazine synthase gene cluster ( $P$. chlororaphis-PHZ) and phenazine biosynthetic pathway (HT66 $\Delta p h z E$ ) (Figure 3). The transcriptional level of $p h z C$ was $29 \%$ decreased after deleting $p h z E$ gene. Thus, it was supposed that changes in the transcriptional levels of genes aroF, aro $G$ and $\operatorname{aroH}$ are caused by the inactivation of $p h z C$ gene, which has a significant influence on the carbon flux of the entire shikimate pathway, and the resulting end products are responsible for the transcriptional regulation of $a r o F, a r o G$ and $a r o H$. Therefore, it was concluded that $p h z C$ is essential for PCN biosynthesis.

\subsection{Mutation and Functional Characterization of DAHP Synthases}

To explore the function of four different DAHP synthases in P. chlororaphis, single DAHP synthase defective strain was constructed through non-scar gene deletion [7]. The derived strains were cultured in KB medium for $72 \mathrm{~h}$. As shown in Figure 4, no morphological difference was found, whereas $p h z C$ mutant strain $\mathrm{HT} 66 \Delta p h z \mathrm{C}$ displayed a reduction in PCN production. The cell growth and PCN titer were checked in KB medium after $84 \mathrm{~h}$ of incubation to further analyze $\mathrm{PCN}$ production. Figure $5 \mathrm{~A}$ showed no significant differences in the cell growth of mutant strains of genes $p h z C$, aroF, aroG and aroH. Surprisingly, the production of PCN was dramatically decreased by the inactivation $p h z C$ gene, but the effect of aroF, aro $\mathrm{G}$ and $\operatorname{aroH}$ genes was less efficient on $\mathrm{PCN}$ production (Figure 5A). The production of PCN was $24.6 \mathrm{mg} / \mathrm{L}, 357.1 \mathrm{mg} / \mathrm{L}, 396.8 \mathrm{mg} / \mathrm{L}$ and $380.5 \mathrm{mg} / \mathrm{L}$ on the single deletion of $\mathrm{phzC}$, aroF, aro $\mathrm{G}$ and aroH respectively. After $36 \mathrm{~h}$ of cultivation, four mutants produced $5.8 \%, 84.2 \%, 96.6 \%$, and $89.7 \%$ of the PCN concentration compared with the wild-type strain, respectively. Consequently, the $\mathrm{PCN}$ production was significantly reduced by knocking out $p h z C$. No significant titer of aromatic amino acids was detected (data not shown). 


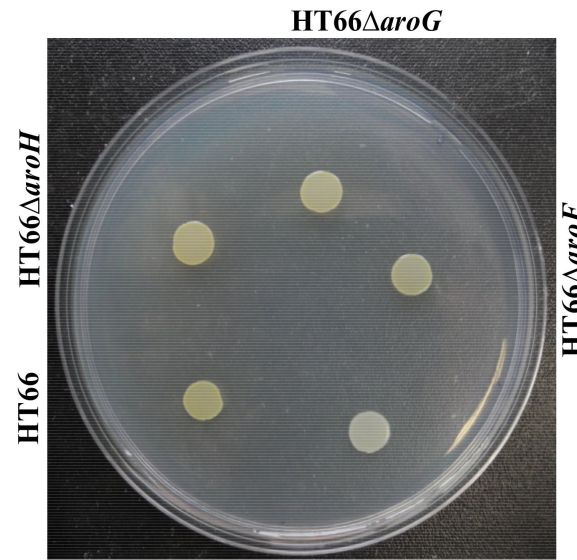

(A) $\mathrm{HT} 66 \Delta p h z C$

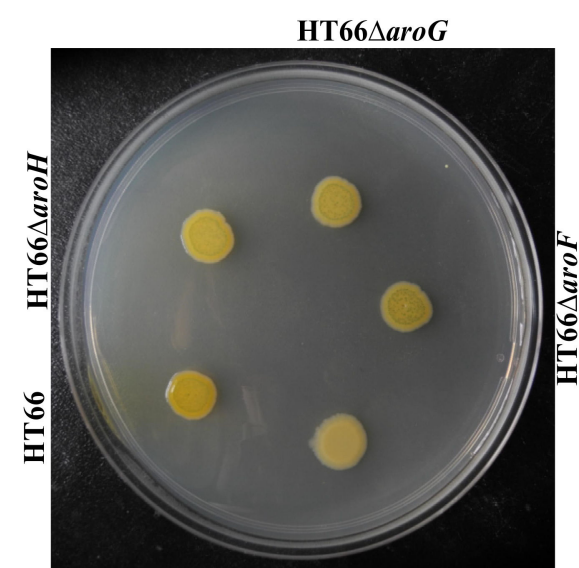

(B) $\mathrm{HT} 6 \mathrm{~b} \Delta \mathrm{phz} \mathrm{C}$

Figure 4. Photographs of the different derivatives grown on KB medium. (A) $24 \mathrm{~h}$; (B) $48 \mathrm{~h}$.

(A)

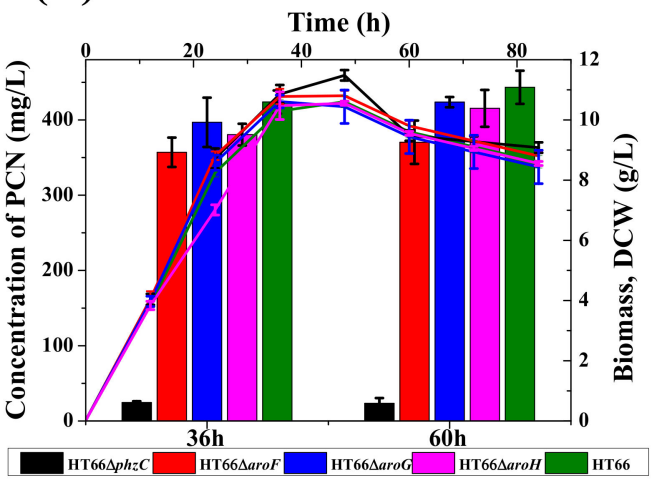

(B)

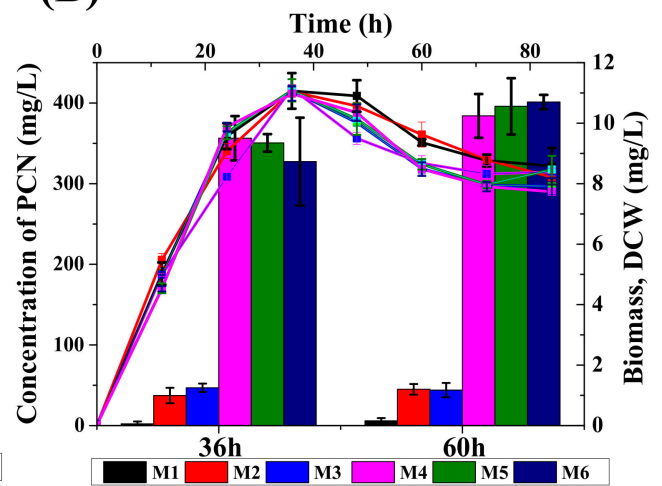

Figure 5. Characteristics growth profile and PCN synthesis of different DAHP synthase-deficient strains. (A) Single-deletion, (B) Dual deletion. M1: HT66 phzC $\Delta a r o F, ~ M 2: ~ H T 66 \Delta p h z C \Delta a r o G$, M3:

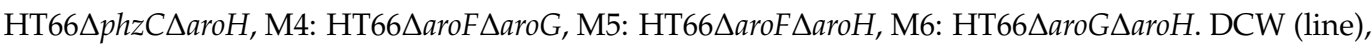
Concentration of PCN (column).

To further investigate the effect of $p h z C$ on PCN production, double DAHP synthases genes were knocked out by constructing six individual mutant strains. Both the cell growth and PCN production were assessed in KB medium, as shown in Figure 5B. Results indicated no significant difference in cell growth among all the derivatives strains. HPLC analysis showed that the PCN production was dramatically decreased to $2.3 \mathrm{mg} / \mathrm{L}, 37.3 \mathrm{mg} / \mathrm{L}$ and

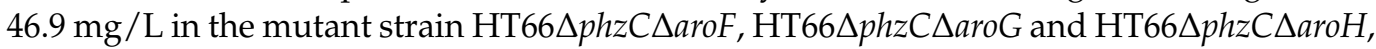
compared to $0.54 \%, 8.8 \%$, and $9.93 \%$ of the $\mathrm{PCN}$ produced by the wild-type strain, respectively. The inactivation of gene $p h z C$ showed that $p h z C$ is essential for PCN biosynthesis. However, the PCN production was decreased by $84 \%, 82.5 \%$, and $77.1 \%$ in the double

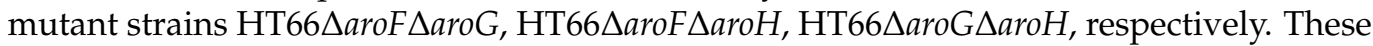
results were consistent with the mutant strains with aroF, aroG and aroH knockout, which showed less significant function in PCN biosynthesis, as revealed by a single gene DAHP synthase inactivation. In the absence of $p h z C, \mathrm{PCN}$ production was decreased by more than $90 \%$, while the inactivation of genes aroF, aro $\mathrm{G}$ and $\operatorname{aroH}$ had a $15 \%$ or less significant effect on $\mathrm{PCN}$ production. In addition, the loss of $\mathrm{phzC}$ reduces the flow of carbon fluxes in the shikimate pathway leading to a substantial reduction in PCN production in P. chlororaphis.

\subsection{Gene Expression Level and Its Quantification}

To better understand the difference among the AroF, AroG, AroH and PhzC in PCN biosynthesis, the transcriptional level of DAHP synthases encoding genes was measured 
after culturing for $26 \mathrm{~h}$. As shown in Figure 6A, the absolute transcriptional level of phzC was normalized to 8.4, higher than aroF (8.2) and aroG (7.9), lower than aroH (8.7), so no significant expression difference was found in the derived strains of $P$. chlororaphis HT66 (Tables S2 and S3 in Supplementary File). It reveals that gene phzC is essential for PCN biosynthesis, which is not dependent on its higher expression level. Moreover, PhzE is the first enzyme that catalyzes chorismate for phenazine derivative synthesis. We also determined the absolute transcript level of gene $p h z E$ in the wild-type strain and found that the transcription level of $p h z C$ was significantly higher than $p h z E$ (Figure 6A). It was supposed that phzC predominantly determines the efficiency of the shikimate pathway, and its high transcriptional level may be involved in the shikimate pathway productivity.

(A)

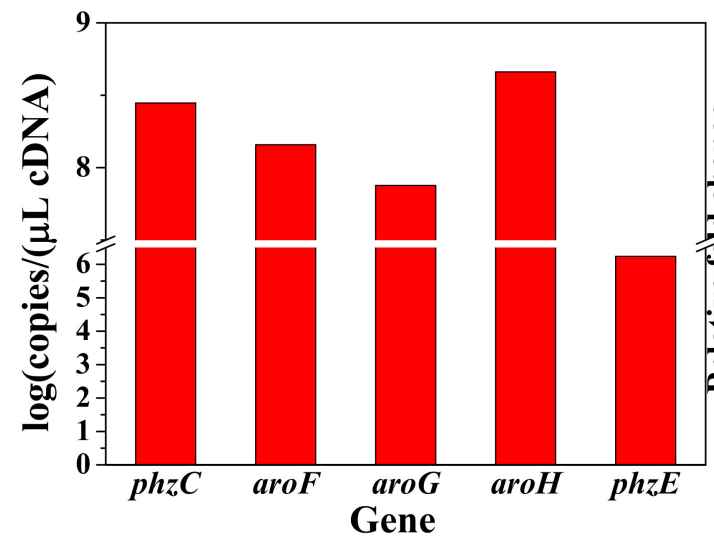

(B)

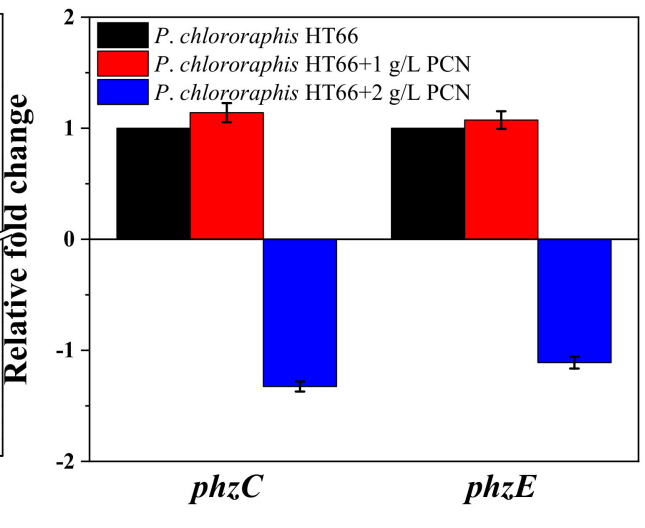

Figure 6. Transcriptional level of different DAHP synthase genes and inhibition of PCN on phzC and phzE transcription. (A) Based on absolute quantification; (B) Based on relative quantification. Calculation of the copy numbers of target gene was shown in Table S4 in Supplementary File.

As the previous studies reported, the transcriptional level of aroF, aroG and aroH were inhibited by aromatic amino acids in E. coli. In this study, the feedback inhibition of genes $p h z C$ and $p h z E$ was studied in strain P. chlororaphis HT66. Strain P. chlororaphis HT66 was cultured in KB medium for $12 \mathrm{~h}$, and PCN was added to the medium at a final concentration of $1 \mathrm{~g} / \mathrm{L}$ and $2 \mathrm{~g} / \mathrm{L}$, respectively. After $26 \mathrm{~h}$ of culturing, the transcriptional levels of genes $p h z C$ and $p h z E$ were determined. After adding different concentrations of PCN, the transcription level of $p h z C$ was up-regulated by 1.14 times (PCN, $1 \mathrm{~g} / \mathrm{L}$ ) and down-regulated by 1.32 times (PCN, $2 \mathrm{~g} / \mathrm{L}$ ), and no significant feedback inhibition was observed (Figure 6B). Therefore, it is speculated that PhzC and PhzE in P. chlororaphis HT66 were not sensitive to feedback inhibition by PCN (pathway end-product).

\subsection{Effect of DAHP Synthase Gene Complementation in the Mutant Strains}

Based on the pBBR1MCS-2 plasmid and the previously screened native $\mathrm{P}_{\text {phz }}$ promoter, phz $\mathrm{C}$, aroF, aroG and aroH were overexpressed and restored into the corresponding single DAHP synthase deficient strain, and the empty plasmid was expressed in the wild type

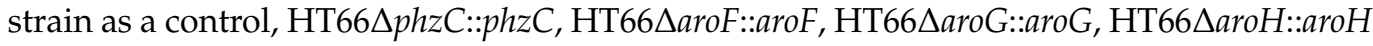
and HT66::pBBR were constructed respectively. The fermentation process was carried out in the shake flask containing KB medium, and PCN production was measured after $36 \mathrm{~h}$ (Figure 7). PCN production was restored to $318.2 \mathrm{mg} / \mathrm{L}$ when $\mathrm{phzC}$ was overexpressed in the $p h z C$ defective strain, which was $78 \%$ and $74.9 \%$ compared with HT66::pBBR and wild type strain, respectively. It is also shown that expressing an empty plasmid pBBRMCS2 in P. chlororaphis HT66 had a slight effect on PCN production, while our previous study reported that antibiotics and inducers displayed a notable effect on the growth of P. chlororaphis [7]. 


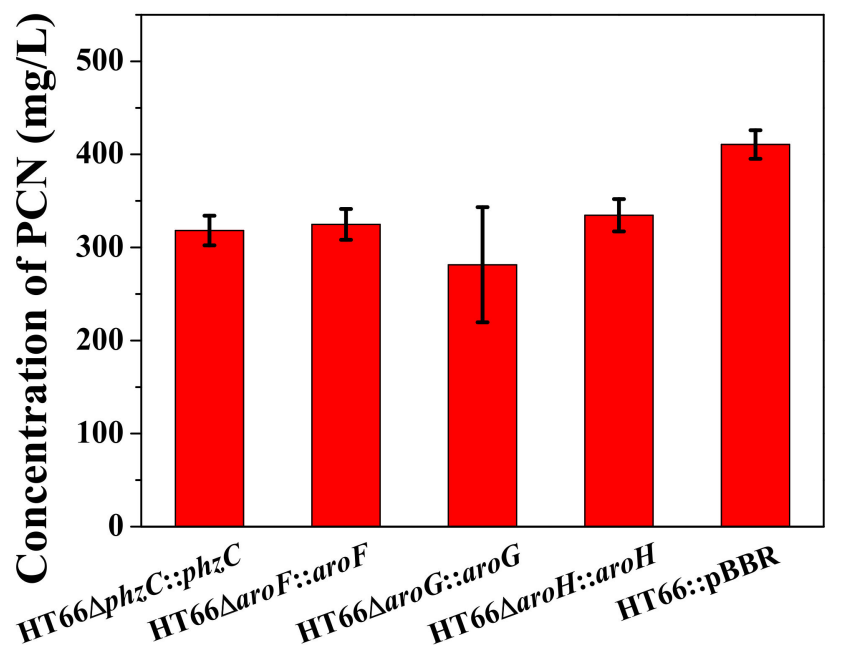

Figure 7. The production of PCN in different DAHP synthase overexpression strains.

\subsection{Effect of DAHP Synthases on the Metabolism of Carbon and Nitrogen Sources}

PhzC catalyzes the condensation reaction of PEP and E4P to produce metabolite DAHP and is essential for PCN biosynthesis in P. chlororaphis HT66. To investigate the effect of DAHP synthases on the carbon metabolism in P. chlororaphis, strains HT66 $\Delta p h z C$, HT66 6 aroF, HT66 $\triangle a r o G$ and HT66 $\triangle a r o H$ were incubated in a medium (MM+glycerol) containing glycerol as the sole carbon source. Results showed no significant difference in colony morphology and the cell growth among different DAHP synthase-deficient strains (Figure 8A). It is indicated that the absence of different DAHP synthase does not affect glycerol utilization.

(A)

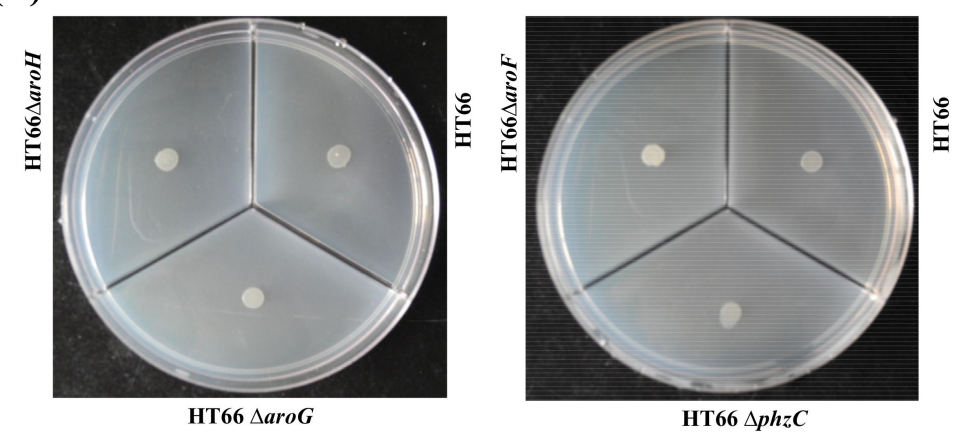

(B)

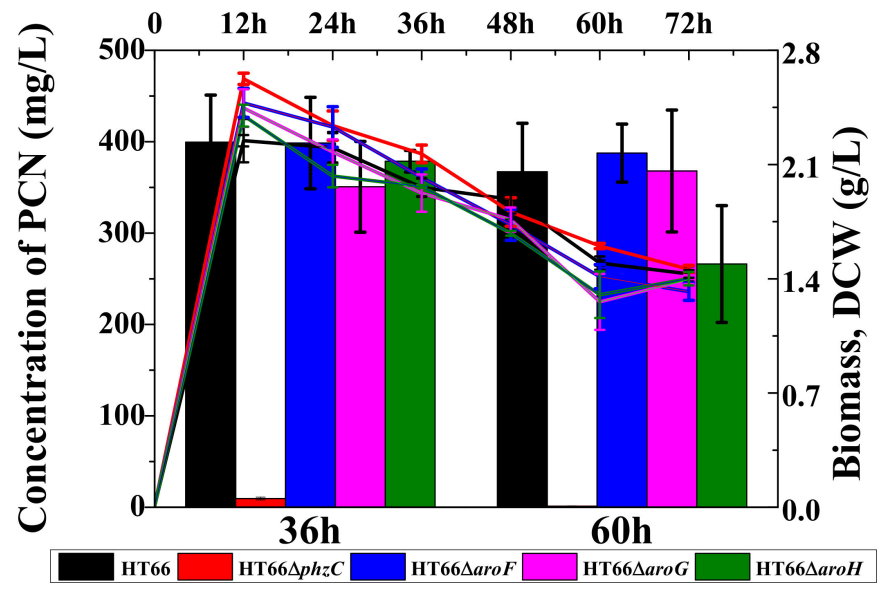

Figure 8. Photographs of $P$. chlororaphis HT66 derivatives grown on specific medium. (A) KB medium without tryptone, (B) KB medium without glycerol. 
As shown in Figure 8B, when the strains HT66, HT66 2 phzC, HT66 2 aroF, HT66 6 aroG and $\mathrm{HT} 66 \mathrm{a}$ aroH were grown in glycerol deficient medium, no significant reduction in PCN production was observed, but the absence of glycerol in the medium reduced the maximum biomass significantly $\left(\mathrm{OD}_{600}\right.$ only reached 6.4). After culturing for $36 \mathrm{~h}$, the concentration

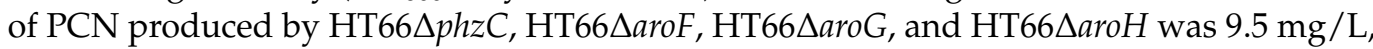
$398.5 \mathrm{mg} / \mathrm{L}, 350.5 \mathrm{mg} / \mathrm{L}$ and $378.7 \mathrm{mg} / \mathrm{L}$, respectively. No significant difference for PCN production was observed in the KB medium. Moreover, P. chlororaphis HT66 could produce $399.5 \mathrm{mg} / \mathrm{L}$ of PCN without glycerol, 10\% less than that harvested in KB medium. The results indicated that gene $p h z C$ displayed no effect on the metabolism of carbon and nitrogen sources. Thus, combining with our early study, it is found that P. chlororaphis HT66 utilizes tryptone as a priority nutrient and then enters glycerol metabolism.

\section{Discussion}

Recently, DAHP synthases were grouped into two distinct classes based on protein sequence homology. The NCBI blasting analysis of DAHP synthase encoding genes shows that, in addition to genes aroF, aroG and aroH, P. chlororaphis HT66 also harbor another gene $p h z C$ encoding DAHP synthase. In E. coli, AroG accounts for approximately $80 \%$ of the total DAHP synthase activity exhibiting a higher specific proteolytic resistance than other DAHP synthases $[39,40]$. However, $p h z C$ was deemed about $90 \%$ of the total DAHP synthase activity for PCN synthesis in P. chlororaphis HT66. Once phzC was inactivated, the production of PCN decreased by more than $90 \%$, indicating that the PhzC is mainly responsible for DAHP synthase activity.

In addition, aroF, aroG and aroH have been widely studied in E. coli and are sensitive to feedback inhibition to aromatic amino acids, tyrosine, phenylalanine and tryptophan, respectively [41-43]. In contrast, by adding different concentrations of PCN to the culture medium, it was found that gene $p h z C$ was not sensitive to feedback inhibition to the pathway end products PCN. Moreover, no feedback inhibition was observed when feeding aromatic amino acids (data not shown). The previous studies have found that phzC in $P$. fluorescens $2-79$ is not conserved in different phenazine producing strains. Unlike aroF, aroG and aroH, phzC lacks an allosteric control loop region and is not sensitive to metabolites feedback inhibition [21]. When using the swiss-model to perform homology modeling, as seen in Figure 9, the affinity of PhzC with PEP and E4P are $-5.9 \mathrm{kcal} / \mathrm{mol}$ and $-5.4 \mathrm{kcal} / \mathrm{mol}$, respectively. The affinity of AroF with PEP and E4P are $-6.6 \mathrm{kcal} / \mathrm{mol}$ and $-5.7 \mathrm{kcal} / \mathrm{mol}$, respectively. The affinity of AroG with PEP and E4P are $-5.2 \mathrm{kcal} / \mathrm{mol}$ and $-4.6 \mathrm{kcal} / \mathrm{mol}$, respectively. The affinity of AroH with PEP and E4P are $-6.6 \mathrm{kcal} / \mathrm{mol}$ and $-5.9 \mathrm{kcal} / \mathrm{mol}$, respectively (Figure 9 ).

DAHP synthase catalyzes the condensation of E4P and PEP to produce DAHP, which is a key step in determining the yield and production of shikimate pathway-based derivatives. Our study found that the loss of different DAHP synthase in P. chlororaphis HT66 did not affect the utilization of carbon sources, such as glycerol. Furthermore, chorismate is converted to ADIC by the biocatalysis of PhzE, and introduces a nitrogen atom through glutamate amino acid, similar to the anthranilate synthase [44]. The PhzF catalyzes the isomerization of DHHA by obtaining a proton from the C3 position of DHHA using a molecule of glutamic acid. In addition, PhzB promotes the formation of diimine hexahydro-phenazine-1,6-dicarboxylic acid (HHPDC) by a two-step catalytic reaction, which is catalyzed by glutamic acid and a pair of histidine/serine, respectively [45]. Amino acids play an essential role in the synthesis of phenazine compounds. When glycerol or tryptone was used as the sole carbon or nitrogen source, different DAHP synthase deficient strains utilized glycerol and tryptone actively. The loss of DAHP synthases did not affect the utilization of carbon and nitrogen sources in P. chlororaphis HT66. At the same time, only tryptone acted as the sole energy source in wild-type strain HT66 resulting in satisfactory production of PCN. In the case of P3 (Derivative strain of P. chlororaphis HT66) strain [46], glycerol is the main carbon source responsible for the high yield of PCN. The above results 
indicated that the carbon and nitrogen-fed fermentation could further increase the yield of the shikimate pathway compound.

(A)

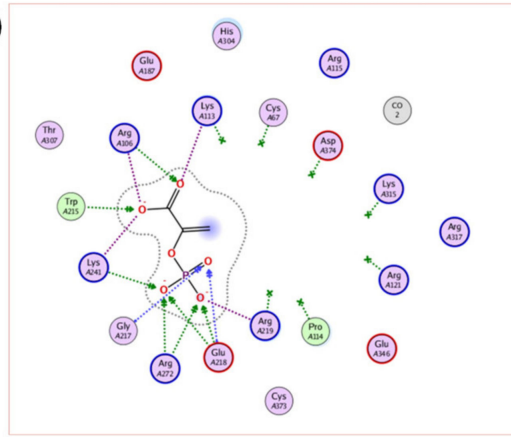

PhzC-PEP

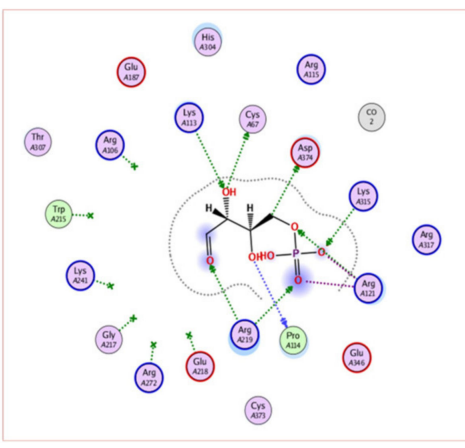

PhzC-E4P

(B)

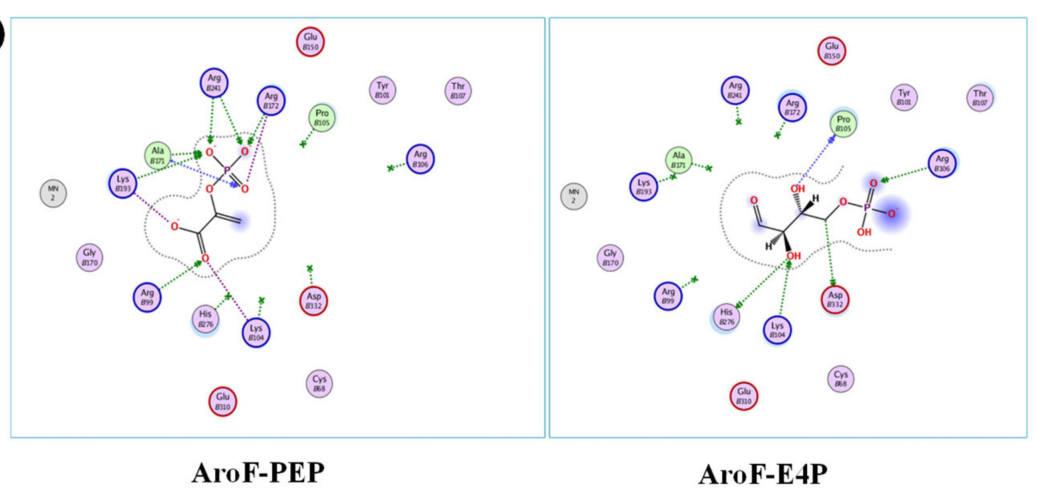

(C)

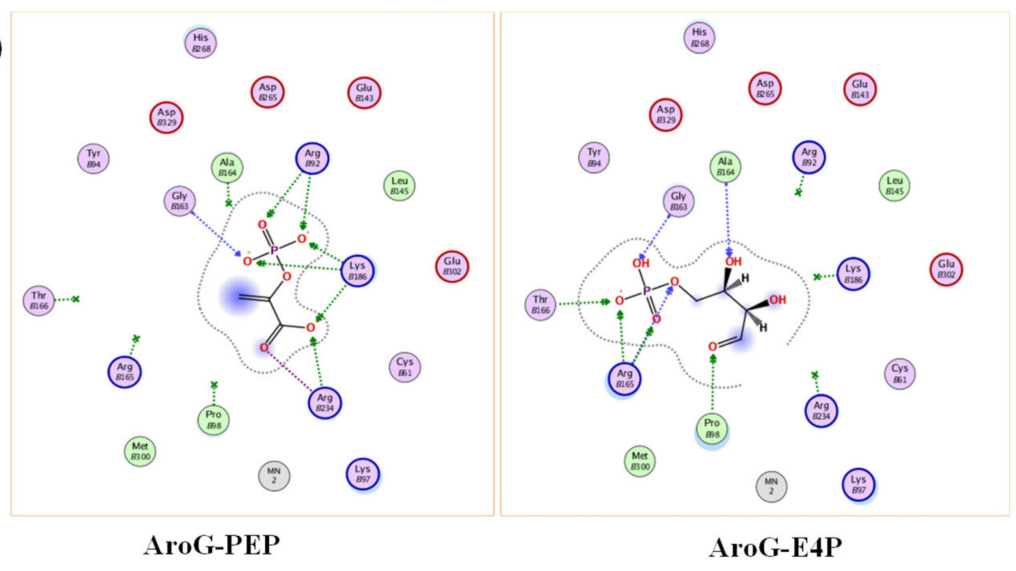

(D)

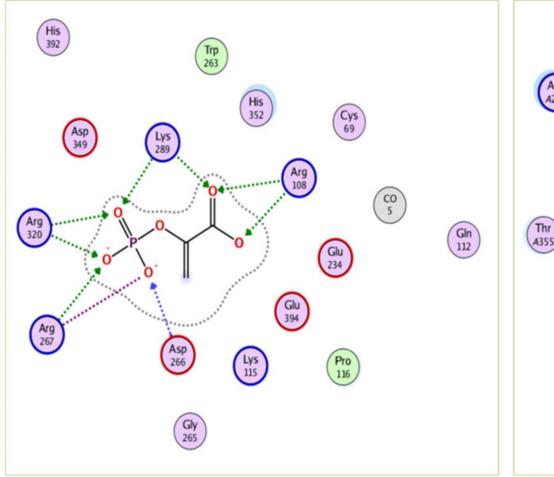

AroH-PEP

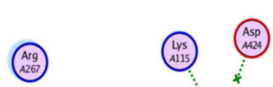

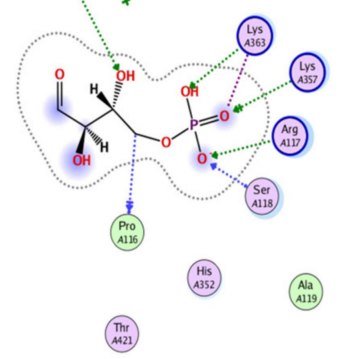

AroH-E4P

Figure 9. Molecular docking of DAHP synthases in P. chlororaphis. Analysis of the binding mode of PEP and E4P with PhzC (A), AroF (B), AroG (C), AroH (D). 
It has been revealed that the dominant role of gene $p h z C$ in the biosynthesis of $P C N$ in $P$. chlororaphis HT66 was not from the difference in transcription levels, but it can be assumed that $p h z C$ mainly determines the carbon flux of the shikimate pathway and its expression is not sensitive to feedback inhibition to the pathway metabolites. These results will promote the construction of the $P$. chlororaphis HT66 cell factory based on the derivatives of the shikimate pathway.

\section{Conclusions}

In this study, we identified and characterized DAHP synthase encoding gene phzC, which differs from the reported DAHP synthases aroF, aroG and aroH in E. coli. It was proved that $\mathrm{PhzC}$ is essential for PCN biosynthesis in the strain P. chlororaphis HT66. Inactivation of gene $\mathrm{phz} C$ resulted in the reduction of $\mathrm{PCN}$ yield by more than $90 \%$, while the absence of genes aroF, aroG and aroH reduced PCN yield by less than 15\%. After the complementation of gene $p h z C$ in the corresponding DAHP synthase-deficient strain, the production of PCN was significantly restored. PhzC has a significant effect on PCN biosynthesis, and its role on the carbon flux of the shikimate pathway in $P$. chlororaphis HT66 is more robust than that of AroF, AroG and AroH. Moreover, the results showed that the gene PhzC in P. chlororaphis HT66 is not sensitive to feedback inhibition and proved that production of high value-added compounds based on the shikimate pathway is feasible and advantageous in P. chlororaphis HT66 cell factory.

Supplementary Materials: The following are available online at https:/ / www.mdpi.com/article/10 .3390/biology11010086/s1, Table S1: Comparative analysis of PhzC in PCN producing and Non-PCN producing strains, Table S2: The original data of relative quantification PCR, Table S3: The original data of absolutequantification PCR, Table S4: Calculation of the copy numbers of target gene.

Author Contributions: S.W. and X.Z. conceived and designed the experiments. S.W. performed experiments, analyzed the experimental data, and drafted the manuscript. D.L., M.B. and W.W. contributed to reagents and materials. X.Z. revised the manuscript. All authors contributed to the final paper. All authors have read and agreed to the published version of the manuscript.

Funding: This work was supported by the National Key Scientific Research Projects (2019YFA0904300) and the National Natural Science Foundation of China (No. 32070051).

Institutional Review Board Statement: Not applicable.

Informed Consent Statement: Not applicable.

Data Availability Statement: Not applicable.

Acknowledgments: X.Z. gratefully acknowledge funding support from the the National Key Scientific Research Projects and the National Natural Science Foundation of China. We are grateful to the Instrumental Analysis Center of Shanghai Jiao Tong University for their skillful technical assistance in LC-MS analysis.

Conflicts of Interest: The authors declare no conflict of interest.

\section{References}

1. Herrmann, K.M.; Weaver, L.M. The Shikimate Pathway. Annu. Rev. Plant Biol. 1999, 50, 473-503. [CrossRef] [PubMed]

2. Averesch, N.J.H.; Krömer, J.O. Metabolic Engineering of the Shikimate Pathway for Production of Aromatics and Derived Compounds-Present and Future Strain Construction Strategies. Front. Bioeng. Biotechnol. 2018, 6, 32. [CrossRef]

3. Floss, H.G. The Shikimate Pathway; Springer: Berlin/Heidelberg, Germany, 1979.

4. Bentley, R.; Haslam, E. The Shikimate Pathway-A Metabolic Tree with Many Branche. Crit. Rev. Biochem. Mol. Biol. 1990, 25, 307-384. [CrossRef] [PubMed]

5. Ikeda, M. Towards bacterial strains overproducing L-tryptophan and other aromatics by metabolic engineering. Appl. Microbiol. Biotechnol. 2006, 69, 615-626. [CrossRef]

6. Noda, S.; Shirai, T.; Oyama, S.; Kondo, A. Metabolic design of a platform Escherichia coli strain producing various chorismate derivatives. Metab. Eng. 2016, 33, 119-129. [CrossRef] [PubMed]

7. Wang, S.; Bilal, M.; Zong, Y.; Hu, H.; Wang, W.; Zhang, X. Development of a Plasmid-Free Biosynthetic Pathway for Enhanced Muconic Acid Production in Pseudomonas chlororaphis HT66. ACS Synth. Biol. 2018, 7, 1131-1142. [CrossRef] 
8. Thykaer, J.; Nielsen, J.; Wohlleben, W.; Weber, T.; Gutknecht, M.; Lantz, A.E.; Stegmann, E. Increased glycopeptide production after overexpression of shikimate pathway genes being part of the balhimycin biosynthetic gene cluster. Metab. Eng. 2010, 12, 455-461. [CrossRef]

9. Pontrelli, S.; Chiu, T.-Y.; Lan, E.I.; Chen, F.Y.-H.; Chang, P.; Liao, J.C. Escherichia coli as a host for metabolic engineering. Metab. Eng. 2018, 50, 16-46. [CrossRef]

10. Contesini, F.J.; de Melo, R.R.; Sato, H.H. An overview of Bacillus proteases: From production to application. Crit. Rev. Biotechnol. 2018, 38, 321-334. [CrossRef] [PubMed]

11. Becker, J.; Wittmann, C. Bio-based production of chemicals, materials and fuels-Corynebacterium glutamicum as versatile cell factory. Curr. Opin. Biotechnol. 2012, 23, 631-640. [CrossRef]

12. Thomas, C.M.; Haines, A.S.; Kosheleva, I.A.; Boronin, A. Pseudomonas: Model Organism, Pathogen, Cell Factory; Wiley-VCH Verlag $\mathrm{GmbH} \& \mathrm{Co} . \mathrm{KGaA}$ : Weinheim, Germany, 2008.

13. Nielsen, J. Yeast cell factories on the horizon. Science 2015, 349, 1050-1051. [CrossRef]

14. Kitade, Y.; Hashimoto, R.; Suda, M.; Hiraga, K.; Inui, M. Production of 4-Hydroxybenzoic Acid by an Aerobic Growth-Arrested Bioprocess Using Metabolically Engineered Corynebacterium glutamicum. Appl. Environ. Microbiol. 2018, 84, e02587-17. [CrossRef]

15. Sun, X.; Lin, Y.; Huang, Q.; Yuan, Q.; Yan, Y. A Novel Muconic Acid Biosynthesis Approach by Shunting Tryptophan Biosynthesis via Anthranilate. Appl. Environ. Microbiol. 2013, 79, 4024-4030. [CrossRef]

16. Shen, X.; Wang, J.; Wang, J.; Chen, Z.; Yuan, Q.; Yan, Y. High-level De novo biosynthesis of arbutin in engineered Escherichia coli. Metab. Eng. 2017, 42, 52-58. [CrossRef] [PubMed]

17. Noda, S.; Shirai, T.; Mori, Y.; Oyama, S.; Kondo, A. Engineering a synthetic pathway for maleate in Escherichia coli. Nat. Commun. 2017, 8, 1-13. [CrossRef] [PubMed]

18. Luo, Z.W.; Kim, W.J.; Lee, S.Y. Metabolic Engineering of Escherichia coli for Efficient Production of 2-Pyrone-4,6-dicarboxylic Acid from Glucose. ACS Synth. Biol. 2018, 7, 2296-2307. [CrossRef]

19. Jin, K.; Zhou, L.; Jiang, H.; Sun, S.; Fang, Y.; Liu, J.; Zhang, X.; He, Y.-W. Engineering the central biosynthetic and secondary metabolic pathways of Pseudomonas aeruginosa strain PA1201 to improve phenazine-1-carboxylic acid production. Metab. Eng. 2015, 32, 30-38. [CrossRef] [PubMed]

20. Mcdonald, M.; Mavrodi, D.V.; Thomashow, L.S.; Floss, H.G. Phenazine biosynthesis in Pseudomonas fluorescens: Branchpoint from the primary shikimate biosynthetic pathway and role of phenazine-1,6-dicarboxylic acid. J. Am. Chem. Soc. 2001, 123, 9459-9460. [CrossRef]

21. Webby, C.J.; Baker, H.M.; Lott, J.S.; Baker, E.N.; Parker, E.J. The Structure of 3-Deoxy-D-arabino-heptulosonate 7-phosphate Synthase from Mycobacterium tuberculosis Reveals a Common Catalytic Scaffold and Ancestry for Type I and Type II Enzymes. J. Mol. Biol. 2005, 354, 927-939. [CrossRef] [PubMed]

22. Walker, G.E.; Dunbar, B.; Hunter, L.S.; Nimmo, H.G.; Coggins, J.R. Evidence for a novel class of microbial 3-deoxy-D-arabinoheptulosonate-7-phosphate synthase in Streptomyces coelicolor A3(2), Streptomyces rimosus and Neurospora crassa. Microbiology 1996, 142, 1973-1982. [CrossRef]

23. Görlach, J.; Beck, A.; Henstrand, J.M.; Handa, A.K.; Herrmann, K.M.; Schmid, J.; Amrhein, N. Differential expression of tomato (Lycopersicon esculentum L.) genes encoding shikimate pathway isoenzymes. I. 3-Deoxy-D-arabino-heptulosonate 7-phosphate synthase. Plant Mol. Biol. 1993, 23, 697-706. [CrossRef] [PubMed]

24. Keith, B.; Dong, X.N.; Ausubel, F.M.; Fink, G.R. Differential induction of 3-deoxy-D-arabino-heptulosonate 7-phosphate synthase genes in Arabidopsis thaliana by wounding and pathogenic attack. Proc. Natl. Acad. Sci. USA 1991, 88, 8821-8825. [CrossRef] [PubMed]

25. Zhao, J.; Herrmann, K.M. Cloning and Sequencing of a Second cDNA Encoding 3-Deoxy-d-arabino-Heptulosonate 7-Phosphate Synthase from Solanum tuberosum L. Plant Physiol. 1992, 100, 1075-1076. [CrossRef]

26. Biessy, A.; Filion, M. Phenazines in plant-beneficial Pseudomonas spp.: Biosynthesis, regulation, function and genomics. Environ. Microbiol. 2018, 20, 3905-3917. [CrossRef]

27. Pierson, L.S., III; Gaffney, T.; Lam, S.; Gong, F. Molecular analysis of genes encoding phenazine biosynthesis in the biological control bacterium Pseudomonas aureofaciens 30-84. FEMS Microbiol. Lett. 1995, 134, 299-307. [CrossRef]

28. Wang, S.; Cui, J.; Bilal, M.; Hu, H.; Wang, W.; Zhang, X. Pseudomonas spp. as cell factories (MCFs) for value-added products: From rational design to industrial applications. Crit. Rev. Biotechnol. 2020, 40, 1232-1249. [CrossRef]

29. Waterhouse, A.; Bertoni, M.; Bienert, S.; Studer, G.; Tauriello, G.; Gumienny, R.; Heer, F.T.; de Beer, T.A.P.; Rempfer, C.; Bordoli, L.; et al. SWISS-MODEL: Homology modelling of protein structures and complexes. Nucleic Acids Res. 2018, 46, W296-W303. [CrossRef]

30. Bienert, S.; Waterhouse, A.; de Beer, T.A.P.; Tauriello, G.; Studer, G.; Bordoli, L.; Schwede, T. The SWISS-MODEL Repository-New features and functionality. Nucleic Acids Res. 2017, 45, D313-D319. [CrossRef]

31. Camacho, C.; Coulouris, G.; Avagyan, V.; Ma, N.; Papadopoulos, J.; Bealer, K.; Madden, T.L. BLAST+: Architecture and applications. BMC Bioinform. 2009, 10, 421. [CrossRef]

32. Remmert, M.; Biegert, A.; Hauser, A.; Söding, J. HHblits: Lightning-fast iterative protein sequence searching by HMM-HMM alignment. Nat. Methods 2011, 9, 173-175. [CrossRef] [PubMed]

33. Morris, G.M.; Huey, R.; Lindstrom, W.; Sanner, M.F.; Belew, R.K.; Goodsell, D.S.; Olson, A.J. AutoDock4 and AutoDockTools4: Automated docking with selective receptor flexibility. J. Comput. Chem. 2009, 30, 2785-2791. [CrossRef] [PubMed] 
34. Shumilin, I.A.; Bauerle, R.; Wu, J.; Woodard, R.W.; Kretsinger, R.H. Crystal Structure of the Reaction Complex of 3-Deoxy-darabino-heptulosonate-7-phosphate Synthase from Thermotoga maritima Refines the Catalytic Mechanism and Indicates a New Mechanism of Allosteric Regulation. J. Mol. Biol. 2004, 341, 455-466. [CrossRef] [PubMed]

35. Livak, K.J.; Schmittgen, T.D. Analysis of Relative Gene Expression Data Using Real-Time Quantitative PCR and the 2- $\Delta \Delta C \mathrm{~T}$ Method. Methods 2001, 25, 402-408. [CrossRef]

36. Ayilavarapu, S.; Doctor, A.; Lee, C.-T.; Tribble, G.D.; Chiu, Y.; Weltman, R.L.; Angelov, N. Altered human alveolar bone gene expression in type 2 diabetes-A cross-sectional study. J. Periodontal Res. 2021, 1-10. [CrossRef] [PubMed]

37. Haslam, E. Shikimic Acid: Metabolism and Metabolites; Wiley: Chichester, UK; New York, NY, USA, 1993.

38. Jin, X.-J.; Peng, H.-S.; Hu, H.-B.; Huang, X.-Q.; Wang, W.; Zhang, X.-H. iTRAQ-based quantitative proteomic analysis reveals potential factors associated with the enhancement of phenazine-1-carboxamide production in Pseudomonas chlororaphis P3. Sci. Rep. 2016, 6, 27393. [CrossRef]

39. Jiang, P.-H.; Shi, M.; Qian, Z.-K.; Li, N.-J.; Huang, W.-D. Effect of F209S Mutation of Escherichia coli AroG on Resistance to Phenylalanine Feedback Inhibition. Sheng Wu Hua Xue Yu Sheng Wu Wu Li Xue Bao Acta Biochim. Biophys. Sin. 2000, 32, $441-444$.

40. Lin, S.; Liang, R.; Meng, X.; Ouyang, H.; Yan, H.; Wang, Y.; Jones, G.S. Construction and expression of mutagenesis strain of aroG gene from Escherichia coli K-12. Int. J. Biol. Macromol. 2014, 68, 173-177. [CrossRef] [PubMed]

41. Ger, Y.-M.; Chen, S.-L.; Chiang, H.-J.; Shiuan, D. A Single Ser-180 Mutation Desensitizes Feedback Inhibition of the PhenylalanineSensitive 3-Deoxy-D-Arabino-Heptulosonate 7-Phosphate (DAHP) Synthetase in Escherichia coli. J. Biochem. 1994, 116, 986-990. [CrossRef] [PubMed]

42. Xu, J.; Hu, C.; Shen, S.; Wang, W.; Jiang, P.; Huang, W. Requirement of the N-terminus for dimer formation of phenylalaninesensitive 3-deoxy-D-arabino-heptulosonate synthase AroG of Escherichia coli. J. Basic Microbiol. 2004, 44, 400-406. [CrossRef]

43. Munack, S.; Roderer, K.; Ökvist, M.; Kamarauskaite, J.; Sasso, S.; van Eerde, A.; Kast, P.; Krengel, U. Remote Control by Inter-Enzyme Allostery: A Novel Paradigm for Regulation of the Shikimate Pathway. J. Mol. Biol. 2016, 428, 1237-1255. [CrossRef]

44. Li, Q.-A.; Mavrodi, D.V.; Thomashow, L.S.; Rossle, M.; Blankenfeldt, W. Ligand Binding Induces an Ammonia Channel in 2-Amino-2-desoxyisochorismate (ADIC) Synthase PhzE. J. Biol. Chem. 2011, 286, 18213-18221. [CrossRef] [PubMed]

45. Parsons, J.F.; Calabrese, K.; Eisenstein, E.; Ladner, J.E. Structure and Mechanism of Pseudomonas aeruginosa PhzD, an Isochorismatase from the Phenazine Biosynthetic Pathway. Biochemistry 2003, 42, 5684-5693. [CrossRef] [PubMed]

46. Wang, S.; Fu, C.; Liu, K.; Cui, J.; Hu, H.; Wang, W.; Zhang, X. Engineering a Synthetic Pathway for Gentisate in Pseudomonas Chlororaphis P3. Front. Bioeng. Biotechnol. 2021, 8, 622226. [CrossRef] [PubMed] 